\title{
Correlation of cardiotocography results and perinatal outcome in gestational hypertension
}

\author{
Ghanghoriya $\mathbf{V}^{1}$, Patel $K^{2}$, Raghuwansh $\mathbf{P}^{3}$ \\ ${ }^{1}$ Dr. Vineeta Ghanghoriya, Assistant Professor, ${ }^{2}$ Dr. Kirti Patel, Senior Resident, ${ }^{3}$ Dr. Pratibha Raghuwansh, Assistant \\ Professor, ${ }^{3}$ Post PG; all authors are attached with Department of Obstetrics \& Gynaecology, NSCB Medical College, \\ Jabalpur, MP, India.
}

Corresponding Author: Dr. Kirti Patel, Dr. Akhilesh Patel, House No 399/B, C/O Sushil Kumarjain, Nehru Nagar, Jabalpur, M.P. Email: dr.kirtipate10107@gmail.com

\begin{abstract}
Background: Early recognition of foetal distress in labour is the primary concern for the obstetrician to avoid adverse perinatal outcome. cardiotocograph is a simple non-invasive test that can serve as a screening tool in assessing foetuses of high risk obstetric patients. Use of electronic FHR monitoring during labour can detect hypoxia timely \& unnecessary delay in intervention can be avoided. Methods: Study was conducted at NSCB MCH Jabalpur, Department of obstetrics \& Gynaecology. Total 200 patients selected who were admitted for hypertensive disorder \& were included in the study. NST was used for fetal surveillance from 32 weeks of gestation weekly, biweekly, alternate day \& daily basis depending on the severity of gestation. patients were followed till delivery \& maternal \& foetal outcome recorded \& correlated with CTG findings. Result: Out of 200 patients 107 delivered by LSCS \& most of these cases had non-reactive NST 70/107 \& 93 delivered by Vaginal route \& most of them have reactive NST 71/93. In non-reactive NST group newborns APGAR score at 5 minute $<7$ in 40/92 cases while in reactive NST group it was in 30/102cases. Total 70 new-born admitted in NICU out of which 63 were from nonreactive group \& 7 were from reactive group. Conclusion: CTG can serve as a screening tool in high risk obstetric pregnancy, particularly in preeclampsia where decrease utero-placental circulation cause considerable fetalcompromise. It helps to detect fetal distress already present or likely to develop during labour.
\end{abstract}

Key words: Cardiotocography, Non stress test, Reactive, Non-reactive, Hypertension.

\section{Introduction}

Early recognition of foetal distress in labour is the primary concern for the obstetrician to avoid adverse perinatal outcome. Good understanding of how an individual fetus reacts to the labour stress help the obstetrician to intervene in appropriate manner. To be more specific during labour fetus can repeatedly suffer from oxygen insufficiency, most fetuses cope well and may mount a 'stress response'.

Foetuses with utero-placental insufficiency develop hypoxia during labour that may be acute or sub-acute. Severe hypoxic injuries can lead to neurological injury, cerebral palsy, neonatal encephalopathy and even death. Current perinatal mortality rate of India is $26 / 1000$ live births[1]. However, it may be underestimated because many cases of stillbirth are not reported. Majority

Manuscript received: $6^{\text {th }}$ February 2018

Reviewed: $16^{\text {th }}$ February 2018

Author Corrected: $24^{\text {th }}$ February 2018

Accepted for Publication: $28^{\text {th }}$ February 2018

Obsgyne Review: Journal of Obstetrics and Gynecology of fetal death occur before the onset of labour. The important causes of chronic hypoxia are hypertensive disorder of pregnancy, antepartum haemorrhage, Rh isoimmunization, maternal heart disease and infection [2]. Themain causes of perinatal mortality are infection, perinatal asphyxia, trauma, prematurity and congenital anomalies.

Hypertensive disorders in pregnancy (HDP) remain a major global health problem not only because of the associated high adverse maternal outcomes but also associated with significant perinatal morbidity and mortality. Although most obstetricians worry more about the risk of maternal death in women whose pregnancies are complicated by hypertensive disorders the risk of perinatal death is more daunting. For instance, the risk of maternal death is less than $1 \%$ in severe preeclampsia and whereas that of perinatal death is about $13 \%$. The situation is even worse in eclampsia where the risks of maternal and perinatal deaths occur 


\section{Original Research Article}

in about $5 \%$ and $28 \%$ respectively. There is risk of serious short and long-term complications in the surviving newborns such as the risk of neurodevelopmental deficits especially in poorly resource countries [3]. Possibilities of stillbirth is 21 per 1000 live birth in cases with severe preeclampsia and 9 per 1000 live birth in mild preeclampsia [4].

CTG appears to be a simple non-invasive test that can serve as a screening tool in assessing fetuses of high risk obstetric patients in hospitals/centres with a heavy workload and limited resources. Continuous electronic fetal heart rate monitoring is routine practice in high risk pregnancies [5].

\section{Aims \& objective}

This study was conducted to see the effect of CTG results on perinatal outcome in gestational hypertension.

\section{Methodology}

This prospective observational study was conducted at tertiary obstetric unit of NSCB medical college Jabalpur. 200 Patients admitted with hypertension in pregnancy were included in the study.

Study design-Prospective Observational study

Place of study-NSCB Medical College \& Hospital Jabalpur, Department of obstetrics \& Gynaecology

Inclusion Criteria- Gestational age $>32$ weeks of pregnancy with hypertensive disorder

Exclusion Criteria-Gestational age $<32$ weeks

-Antepartum eclampsia patients

NST was used for fetal surveillance from 32 weeks of gestation weekly, biweekly, alternate day and on daily basis depending on the severity of hypertensive disorder of pregnancy and were followed up. Cardiotocography was done by 'Koddel Cardiotocograph 'equipment applied over the maternal abdomen in left lateral position. The patients BP and pulse were recorded every 20 minutes during the procedure. Patient was instructed to push event marker button every time she felt fetal movement. External tocodynamometer device record the uterine activity by detecting the changes in the surface abdominal wall tension.

The simultaneous recording was traced for 20 minutes and if there was no acceleration meeting the criteria, vibro acoustic stimulation (VAS) was given with an artificial larynx of approximately $80 \mathrm{hz}$ and $82 \mathrm{db}$ for $1-3$ second. The NST was recorded for 20 minutes and extended upto 40 minutes for non-reactive traces.

The NST was classified into 3 groups (NICE)[6]based on the presence or absence of at least 2 FHR accelerations of $15 \mathrm{bpm}$ lasting 15 seconds in a 20 minutes reading into

(1) Reactive or normal test

(2) Non-reactive or abnormal test

(3) Suspiciousor equivocal test. In the suspicious test group NST was done with vibroacoustic stimulation and extended to 40 minutes and the results are further classified as reactive or normal test and nonreactive or abnormal test based on reactivity criteria's.

Patients were followed up for the mode of delivery. At the time of delivery, data of following variables were collected like fetal distress during labour, meconium stained liquor, 1-minute APGAR $<4,5$ minute APGAR $<7$, NICU admission, indication of NICU admission, duration of stay in NICU and perinatal mortality.

\section{Results}

Total 200 subjects were included in the study. NST was more non-reactive in severe preeclampsia cases $53.63 \%(83 / 179)$ than in mild preeclampsia $42.85 \%(9 / 21)$.

Table-1: Correlation of gestational age with reactivity of NST.

\begin{tabular}{|c|c|c|}
\hline \multirow{2}{*}{ Gestational age } & \multicolumn{2}{|c|}{ NST } \\
\cline { 2 - 3 } & Reactive $(\mathbf{n = 1 0 8})$ & Non-reactive (n=92) \\
\hline$<34$ weeks $(\mathrm{n}=15)$ & $5(33.33 \%)$ & $10(66.67 \%)$ \\
\hline $34-37$ weeks $(\mathrm{n}=34)$ & $20(58.82 \%)$ & $14(41.18 \%)$ \\
\hline$>37$ week $(\mathrm{n}=151)$ & $83(54.97 \%)$ & $68(43.03 \%)$ \\
\hline
\end{tabular}

*P Value $<0.05)$. NST shown more reactivity at higher gestational age and higher nonreactivity with lower gestational age (table 1). 
Original Research Article

Table-2: Correlation of reactivity of NST with mode of delivery

\begin{tabular}{|c|c|c|}
\hline \multirow{2}{*}{ NST } & \multicolumn{2}{|c|}{ Delivery mode } \\
\cline { 2 - 3 } & LSCS $(\mathbf{n = 1 0 7})$ & VD(n=93) \\
\hline Reactive $(\mathrm{n}=108)$ & $37(34 \%)$ & $71(66 \%)$ \\
\hline Nonreactive $(\mathrm{n}=92)$ & $70(76 \%)$ & $22(24 \%)$ \\
\hline
\end{tabular}

$* \mathrm{P}$ Value $<0.05$

Maximum cases with nonreactive NST 76\% were delivered by LSCS while maximum cases with reactive NST delivered vaginally $66 \%$.

Table-3: Association of NST with APGAR score at 5 minutes

\begin{tabular}{|c|c|c|}
\hline \multicolumn{2}{|c|}{ APGAR SCORE } & \multicolumn{2}{|c|}{ NST } \\
\cline { 2 - 3 } & Reactive (=108) & Non-reactive (n=92) \\
\hline At 5 minutes normal i.e. $\geq 7(\mathrm{n}=130)$ & $78(72 \%)$ & $52(57 \%)$ \\
\hline At 5 minutes abnormal i.e. $<7(\mathrm{n}=70)$ & $30(28 \%)$ & $40(43 \%)$ \\
\hline
\end{tabular}

P Value $<0.05$

Patients having reactive NST had babies with significantly better APGAR score at 5 min than the babies of patients with non-reactive NST (table 3)

Table-4: Indication of NICU admission \& its correlation with NST.

\begin{tabular}{|c|c|c|}
\hline \multirow{2}{*}{ Indication of NICU admission } & \multicolumn{2}{|c|}{ NST } \\
\cline { 2 - 3 } & Reactive $(\mathbf{n}=7)$ & Non-reactive $(\mathbf{n}=\mathbf{6 3})$ \\
\hline Low birth weight $(\mathrm{n}=15)$ & $4(26.67 \%)$ & $11(73.33 \%)$ \\
\hline Meconium stained liqour $(\mathrm{n}=45)$ & $2(4.44 \%)$ & $43(95.56 \%)$ \\
\hline Respiratory distress $(\mathrm{n}=10)$ & $1(10 \%)$ & $9(90 \%)$ \\
\hline
\end{tabular}

$* \mathrm{P}$ Value $<0.05$

Total 70 babies were admitted in NICU. Out of these $90 \%(n=63)$ were associated with non-reactive NST. NICU admissions were due to meconium aspiration, respiratory distress and low birth weight (table 4)

Table-5: Correlation of NST with survival of new-borns.

\begin{tabular}{|c|c|c|}
\hline \multirow{2}{*}{ NST } & \multicolumn{2}{|c|}{ Condition of babies } \\
\cline { 2 - 3 } & Died (=14) & healthy (n=186) \\
\hline Reactive $(\mathrm{n}=108)$ & $1(1)$ & $107(99 \%)$ \\
\hline Non-reactive $(\mathrm{n}=92)$ & $13(15 \%)$ & $79(85 \%)$ \\
\hline
\end{tabular}

$* \mathrm{P}$ Value $<0.001$

Out of 200 babies 7\% $(n=14)$ were died. mortality was significantly associated with nonreactive NST (table 5)

NST shown more reactivity at higher gestational age and higher nonreactivity with lower gestational age (table 1) Maximum cases with nonreactive NST 76\% were delivered by LSCS while maximum cases with reactive NST delivered vaginally $66 \%$. Patients having reactive NST had babies with significantly better APGAR score at 5 min than the babies of patients with non-reactive NST (table 3). Total 70 babies were admitted in NICU. Out of these 90\%(n=63) were associated with non-reactive NST. NICU admission were due to meconium aspiration, respiratory distress and low birth weight (table 4). Out of 200 babies $7 \%(n=14)$ were died. Mortality was significantly associated with nonreactive NST (table 5). 


\section{Original Research Article}

\section{Discussion}

Use of electronic FHR monitoring during labour can detect hypoxia timely and unnecessary delay in intervention can be avoided. In our study we included 200 patients. Out of these 200 patients 107 patients delivered by LSCS and most of these cases had nonreactive NST. Early identification of non-reactive NST helped in taking decision timely. Sandhu et al had concluded in their study that cardiotopographic testing could be used to identify patients likely to develop adverse fetal outcome and help in optimal utilization of labour room resources[7].

It is a non-invasive recordable screening test for the state of oxygenation of the foetus. It assesses the placental reserve by checking the response of the foetal heart during the phase of temporary occlusion of the utero-placental circulation under physiological stress of repeated uterine contractions.

High risk obstetric patients had more perinatal mortality because fetus is already compromised. Buscicchio et al demonstrated in their study that pregnancies complicated by gestational diseases do an impact on FHR [8].

Non- reactive NST group newborn's APGAR score at 5 min was $<7$ in most of them. APGAR score is a proxy for perinatal asphyxia and indicator of compromised fetus. Backes et al also found that Gestational hypertension is the most important among high risk pregnancies with higher perinatal morbidity and mortality. Gestational hypertension is associated with iatrogenic prematurity, foetal growth restriction and foetal over growth [9].

It is a risk factor for respiratory distress and neonatal hypoglycaemia etc. Pijnenborg found high rate of perinatal asphyxia, and hypoxicischaemic encephalopathy in babies of hypertensive motherdue to the links between imperfect placentation and accelerated placental ageing[10,11]. Barunoday Chakraborty et al found in their study that patients with non-reactive CTG had neonates with abnormal APGAR score [12].

In present study NST reactivity shows positive correlation with gestational age. Higher the gestational age more reactive was NST. Lower gestational age showed predominantly non-reactive NST.Where CTG had proved its effectiveness in reducing the perinatal mortality.
This would approximate to about 1.5-2 million neonatal deaths annually. In present study out of 200 babies 14 (7\%) were died. It was statistically significantly higher in non-reactive NST group $(n=13)$ then in reactive NST group $(n=1)$. It has been estimated that the hypertensive disorders of pregnancy precede $10 \%$ of early neonatal deaths (8/1000 live births)[13] and a significant proportion of late neonatal deaths (3/1000 live births) [14]. Emma R. Lawrence et al in her study found significant decrease $(p=0.01)$ in perinatal mortality following implementation of CTG technology. 17.3\% (44/254) perinatal death in non CTG group and 6.8\% $(8 / 118)$ in CTG group[15].

Total 70 newborn were admitted to NICU out of which 63 were of non-reactive NST group and 7 were of reactive NST group. Maximum admissions were of nonreactive group which is statistically significant. Manisha Gupta et el study shows that the foetuses with reactive CTG required less NICU admission as compared to non reactivefetuses $(22.8 \% \mathrm{v} / \mathrm{s} 75.7 \%)$ with $\mathrm{P}<0.001[16]$.

So NST help us to take timely decision and improve the perinatal outcome in hypertensive patients.

\section{Conclusion}

Results of this study shows that patients with nonreactive NST results had more perinatal morbidity and mortality than those with reactive NST results. CTG can serve as a screening tool in high risk obstetric pregnancies, particularly in pre-eclampsia where decrease utero-placental circulation cause considerable fetal compromise. It helps to detect foetal distress already present or likely to develop during labour and avoid unnecessary delay in intervention.

As the test has high specificity, it has a role in obstetric wards of developing countries with heavy workload with a large number of high-risk cases.

Funding: Nil, Conflict of interest: Nil

Permission from IRB: Yes

\section{References}

1. Registrar General of India. Sample registration system (SRS) statistical report 2013. New Delhi.

2. Damian Hutter, John Kingdom, and Edgar Jaegg. Causes and Mechanisms of Intrauterine Hypoxia and Its Impact on the Fetal Cardio vascular System: A Review. international Journal of Pediatrics. Volume 2010 (2010), Article ID 401323, 9 pages. 


\section{Original Research Article}

3. Kwame Adu-Bonsaffoh, Michael Y. Ntumy, Samuel A. Obed, and Joseph D. Seffah et al. Perinatal outcomes of hypertensive disorders in pregnancy at a tertiary hospital in Ghana. BMC Pregnancy Childbirth. 2017; 17: 388 DOI 10.1186/s12884-1575-2

4. J.R. Barton, L. A. Barton, N. B. Istwan. Elective delivery at 34 to 36 weeks' gestation and its impact on neonatal outcomes in women with stable mild gestational hypertension, American Journal of Obstetrics and Gynecology. 2011; 204:44.e1-5.

5. Trupti J, Dhakare and Kiran P Patole. A Study of Cardiotocography during Active Labour to Assess the Perinatal Outcome in High Risk Pregnancy. MVP Journal of Medical Sciences, 2016; Vol 3(2):92-95.

6. National institute of clinical excellence intrapartum care. Care of health women and their babies during labour. Nice clinical guideline No.55 september 2007.

7. Gp Capt GS Sandhu, VSM, R Raju, Col TK Bhattacharyya, Lt Col Shaktivardhan. Admission Cardiotocography Screening of High Risk Obstetric Patients. MJAFI 2008; 64 : 43-45

8. Buscicchio G, Gentilucci L, Tranquilli AL. Computerized analysis of fetal heart rate in pregnancies complicated by gestational diabetes mellitus, gestational hypertension, intrauterine growth restriction and premature rupture of membranes. $\mathrm{J}$ MaternFetal Neonatal Med. 2010 Apr; 23 (4): 335-7. doi: 10.3109/ 14767050903258712 .

9. Backes $\mathrm{CH}$, Markham K, Moorehead P, Cordero L, Nankervis CA, Giannone PJ. Maternal preeclampsia and neonatal outcomes. J Pregnancy. 2011; 2011: 214365. doi: 10.1155/2011/214365. Epub 2011 Apr 4.

10. PijnenborgR, Anthony J, Davey DA, Rees A, Tiltman A, Vercruysse L, van Assche A. Placental bed spiralarteries in the hypertensive disorders of pregnancy. Br J Obstet Gynaecol. 1991 Jul; 98 (7): 648-55.
11. Roberts JM, Escudero C. The placenta in preeclampsia. Pregnancy Hypertens. 2012 Apr 1;2(2): 72-83.

12. Barunoday Chakraborty, Tamal Kumar Mondal, Sannyasi Charan Barman, Biswa Pratim Rudra, Ramkrishna Sahana, Prabhat Chandra Mondal. Evaluation of Perinatal Outcome by Antenatal CTG and Umbilical Artery Doppler in Pre-eclamptic Mothers. Indian Journal of Clinical Practice.2013; Vol. 24, No. 6.

13. Lawrence E. R., Quarshie E. L., Lewis, K. F. Peltzman, T., \& Anderson F. J. Introduction of cardiotocograph monitoring improves birth outcomes in women with preeclampsia in Ghana. International Journal of Gynecology and Obstetrics. 2016; 132(1), 103-104.

14. Lawn JE, Blencowe H, Waiswa P, Amouzou A, Mathers C, Hogan D, Flenady V, Frøen JF, Qureshi ZU, Calderwood C, Shiekh S, Jassir FB, You D, McClure EM, Mathai M, Cousens S; Lancet Ending Preventable Stillbirths Series study group; Lancet Stillbirth Epidemiology investigator group. Stillbirths: rates, risk factors, and accelerationto wards 2030. Lancet. 2016 Feb 6;387 (10018): 587-603. doi: 10.1016/S0140-6736 (15) 00837-5. Epub 2016 Jan 19.

15. Vogel JP, Souza JP, Mori R, Morisaki N, Lumbiganon P, Laopaiboon M, Ortiz-Panozo E, Hernandez B, Pérez-Cuevas R, Roy M, Mittal S, Cecatti JG, Tunçalp Ö, Gülmezoglu AM; WHO Multicountry Survey on Maternal and Newborn Health Research Network. Maternal complications and perinatal mortality: findings of the World Health Organization Multicountry Survey on Maternal and Newborn Health. BJOG. 2014 Mar;121 Suppl 1:76-88. doi: 10.1111/ 1471-0528.12633.

16. Manisha Gupta, Teena Nagar, Punit Gupta. Role of Cardiotocography to Improve Perinatal Outcome in High Risk Pregnancy. International Journal of Contemporary Medical Research ISSN. Volume 4 | Issue 4 | April 2017;2454-7379 | ICV (2015): 77.83

\section{How to cite this article?}

Ghanghoriya V, Patel K, Raghuwansh P, Post PG. Correlation of cardiotocography results and perinatal outcome in gestational hypertension. Obs Rev:J obstet Gynecol 2018;4(1):1-5.doi: 10.17511/joog.2018.i01.01. 